\title{
Efficient Cost Computation in Cost Map Planning for Non-Circular Robots
}

\author{
Jennifer King \\ Computer and Information Science \\ University of Pennsylvania \\ Philadelphia, PA 19104 \\ jeking@seas. upenn.edu
}

\author{
Maxim Likhachev \\ Computer and Information Science \\ University of Pennsylvania \\ Philadelphia, PA 19104 \\ maximleseas. upenn.edu
}

\begin{abstract}
For a robot with a circular footprint, obstacles in a map can be inflated by the radius of the footprint, and planning can treat the robot as a point robot. Many robotic vehicles however have non-circular footprints. When operating in cluttered spaces it therefore becomes important to evaluate the footprint of these robots against a cost map. This evaluation is one of the major computational burdens in planning for robots whose footprints can not be assumed to be circular.

In this paper, we propose an efficient method for evaluating a footprint of the robot against a cost map. Our method involves a transformation of the set of points covered by the footprint of the robot into two sets of points: points that should be evaluated against the cost map with inflated obstacles, and points that should be evaluated against the original cost map. The cumulative number of these points is much smaller than the number of points in the original footprint of the robot. Moreover, the method automatically reduces the robot to a single point when its footprint is circular. On the theoretical side, the paper proves the correctness of our method. On the experimental side, the paper shows that the method results in a significant speedup.
\end{abstract}

\section{INTRODUCTION}

Fast path planning is an important part of many robotic applications. The ability to quickly compute and recompute paths is imperative for mobile robots moving amongst static and dynamic obstacles. Perhaps, one of the most common approaches to planning is planning with grid-based cost maps [7][8][13]. Grid-based cost maps are simple to construct, are able to represent arbitrary shape obstacles and can incorporate complex cost functions.

When planning for a robot with a circular footprint, all obstacles in the cost map are typically inflated by the radius of the footprint and the representation of the robot is reduced to a point robot [3]. In such cases, the evaluation of a cost of an action reduces to a simple look-up of the costs of the cells traveled by the robot while executing this action. While very effective for circular robots, this optimization is not applicable to the robots with non-circular footprints.

For non-circular robots operating in cluttered environments with narrow passages (e.g., doorways, parking spots), it is important to plan by taking the orientation of the robot into account and using the actual footprint of the robot [10]. Such planning becomes expensive for several reasons. First, it needs to be done in at least 3-dimensions ( $x, y$ and orientation). Second, the evaluation of a cost of an

This work was in part supported by Willow Garage action becomes highly expensive. In fact, usually, this cost evaluation procedure becomes the most time-consuming part of planning: it requires the convolution of the set of all the cells visited by the robot during execution of an action with a cost map. For example, a $1 \mathrm{~m}$ by $1 \mathrm{~m}$ square robot operating in a $2.5 \mathrm{~cm}$ grid covers almost 500 cells for a simple turn-inplace action.

In this paper, we propose an efficient method for calculating action costs. The method is applicable to any ground robots, regardless of their footprints or holonomic constraints. Our method first reduces the number of cells to consider in the cost calculation by removing sets of points from the footprint which fall inside circles of a fixed radius. Then, it computes a second cost map which essentially inflates the original cost map by the radius of the removed circles. When calculating the action cost, the points which do not fall inside the circle are convolved against the original cost map and the circle centers are convolved against the new cost map. The results of these two separate convolutions are combined to create a single action cost. This transformation reduces significantly the number of points involved in the convolution and thus allows for a faster cost calculation.

In case of a circular robot, our method automatically reduces to representing the robot with a single point and inflating the map by the radius of the robot. For non-circular robots, our method provides a provably-correct optimization whose benefit depends on how close the footprint of the robot resembles a circular footprint. In our theoretical analysis, we show the correctness of the proposed method. In our experimental analysis, we show that the method can result in a significant speedup (about three-fold) over the current approaches.

\section{RELATED WORK}

In some approaches to planning, the cost map utilized for computing action cost is binary [2][5]. In such cases, a cell cost is infinite when the cell is occupied by an obstacle and zero otherwise. In these environments, action cost calculation essentially requires the detection of obstaclecollisions during execution of the action. Rather than perform the footprint convolution, collision detection algorithms such as those described in [1][11][12] can be utilized. These typically provide methods which are computationally less expensive. However, they do not generalize well to action 
cost computation in maps with non-binary costs. Additionally, they are often limited to a particular shape or class of obstacles.

Tornero, Hamlin and Keyy suggest that any threedimensional polygon can be represented by an infinitely sized set of spheres, or alternatively, any two-dimensional polygon can be represented by an infinitely sized set of circles. They note that the accuracy of the representation is directly related to the complexity for obtaining these circles[15]. Limiting the set of spheres used in the representation to a single radius can allow the methods applicable to circular robots to generalize.

Previous works have also improved performance of action cost computation for any class of robots by pre-computing the cells covered when executing an action[10]. Additional performance improvements have been obtained by keeping a second cost space map with all obstacles inflated by the radius of the smallest circle completely enclosing the robot. All of the cells through which the center of the robot passes during the action are first checked against this second map. If all of the centers are obstacle free in the second map, the action is guaranteed to be obstacle free. Only those actions that do not produce conclusive results need to be convolved with the cost map. Each of these methods can be used in conjunction with our transformation to further increase efficiency.

\section{Algorithm}

Usually, in cost map planning, the cost of each cell ranges from 0 to $\infty$, where 0 corresponds to a fully traversable cell, $\infty$ corresponds to an obstacle (untraversable), and any finite value in between corresponds to some degree of traversability (or risk or some other cost function)[16]. The cost map planners then compute the cost of an action in the following manner. First, they iterate over all of the cells (points) covered by the footprint of the robot during the execution of the action (several examples are shown in Figure 4, top image in each sub-figure). During this iteration, they compute either the average or the maximum of the costs of the cells they iterate over. If any of these cells contain obstacles, then both the average and the maximum will be equal to $\infty$. Second, the cost of the action is set to be the length of the action (or time it takes to execute it) times the computed average or computed maximum plus one. Thus, the cost of an action that involves the footprint of the robot going over an obstacle becomes infinite, and therefore the whole action becomes invalid. On the other hand, the cost of an action for which the footprint of the robot goes over only fully traversable cells becomes simply the length of the action (or the time it takes to execute it). The first of these steps is highly expensive, and our method is directed towards reducing its expense. In the following, we will assume that in this step we are interested in computing the maximum over the cells.

The basic idea behind our algorithm is to represent the footprint of the robot traversing each action (footprint of the action) with a set of circles of fixed radius $r$, which fit completely inside the footprint (see Figure 4, bottom image in each sub-figure). This modification greatly reduces the number of cells used in the representation of the footprint of the action. Using only circles, however, leaves some cells out. To address this, we introduce the concept of a remainder set. The remainder set is defined as all of the points in the footprint of the action which cannot be enclosed by any of the circles used in our representation. Our new representation of the footprint of an action is therefore defined as the union of two sets of points: the centers of the circles, each of radius $r$ and the remainder set.

In addition to modifying the representation of the footprint of each action, we also require the use of two cost maps. The first map is the original cost map. This map is used to look up the value of the remainder points when performing the action cost computation. The second map is a modified cost map with all the obstacles inflated by the radius $r$ of the circles used in the representation. To achieve this inflation, every cell in the new cost map is defined to be the maximum of all the cells within radius $r$ in the original map. This map is used to calculate the value of each circle center.

Picking an optimal circle radius $r$ and finding the number of circles that minimize the number of the circle centers plus the size of the remainder set is an optimization problem. One approximation to this however can simply be picking $r$ to be the radius of the largest circle that can be inscribed into the footprint of the robot at the center of the robot and computing the centers of the circles for an action to be all the cells visited by the center of the robot while executing the action.

Formal definition of the algorithm In the following, we define the algorithm formally. Let $\left\{m_{1} \ldots m_{N}\right\}$ be a set of cells. Let us also define a mapping, $M_{0}(\cdot)$, which takes a cell and returns a value in between 0 and $\infty$ that represents the cost of that cell in the cost map.

Let us now define the footprint $F$ of an action as a set of cells $\left\{f_{1} \ldots f_{n}\right\}$ where each $f_{i}$ is a cell in $\left\{m_{1} \ldots m_{N}\right\}$. We define the cost of the footprint as follows:

\section{Definition 1: $C_{F}=\max _{f_{i} \in\left\{f_{1} \ldots f_{n}\right\}} M_{0}\left(f_{i}\right)$}

Thus, evaluating the cost of an action involves computing $C_{F}$. Our transformation allows for the computation of $C_{F}$ to be faster than iterating over all the points in $\left\{f_{1} \ldots f_{n}\right\}$. In particular, our method transforms the set $\left\{f_{1} \ldots f_{n}\right\}$ into two sets: set $\left\{c_{1} \ldots c_{q}\right\}$ of $q$ centers of circles, each of radius $r$, and set $\left\{r_{1} \ldots r_{m}\right\}$ of the remaining $m$ cells. In other words, our method transforms the footprint $F$ into a new footprint $F^{\prime}=\left\{c_{1} \ldots c_{q}\right\} \cup\left\{r_{1} \ldots r_{m}\right\}$. The following three definitions formally define this transformation.

Definition 2: Define a new footprint $F^{\prime}=\left\{c_{1} \ldots c_{q}\right\} \cup$ $\left\{r_{1} \ldots r_{m}\right\}$ such that $\forall f_{i} \in F$ one and only one of the following statements hold:

1) $D\left(f_{i}, c_{j}\right) \leq r$ for some $j$ (where $D\left(f_{i}, c_{j}\right)$ defines the distance between $f_{i}$ and $c_{j}$ )

2) $f_{i}=r_{j}$ for some $j$ 


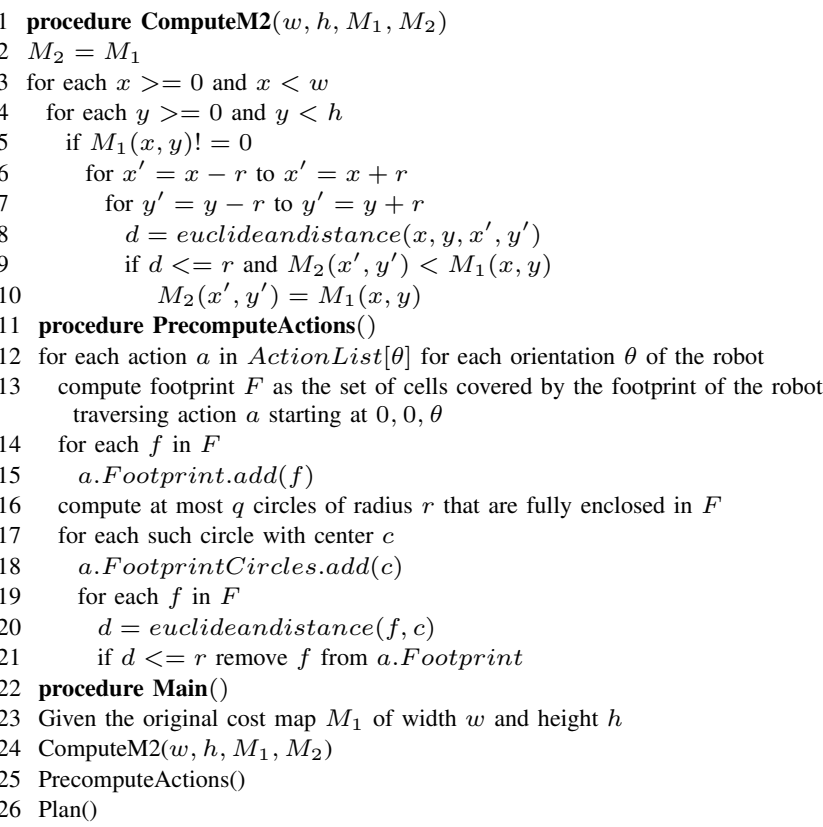

Fig. 1. Footprint Transformation Algorithm

Definition 3: Each circle removed from the footprint must be entirely contained within the footprint. Formally:

$$
\forall i, j \text { s.t. } D\left(m_{i}, c_{j}\right) \leq r \Rightarrow \exists k \text { s.t. } m_{i}=f_{k}
$$

Definition 4: Define two new mappings, $M_{1}(\cdot), M_{2}(\cdot)$ as follows:

1) Mapping $M_{1}$ is an exact copy of $M_{0}$. Formally, $\forall i$ $M_{1}\left(m_{i}\right)=M_{0}\left(m_{i}\right)$.

2) Mapping $M_{2}$ returns the value which is the maximum of all cells in the original map within radius $r$. Formally, $\forall i M_{2}\left(m_{i}\right)=\max _{m_{j} \mid D\left(m_{i}, m_{j}\right) \leq r} M_{0}\left(m_{j}\right)$.

The above definition 4 corresponds to defining two maps. Our method maintains both of these maps, and uses both of them in evaluating the cost of the transformed footprint $F^{\prime}$. The following two definitions define exactly how the cost of the footprint $F^{\prime}$ of an action is computed. First, let us break $F^{\prime}$ into two subsets $F_{1}^{\prime}$ and $F_{2}^{\prime}$, where $F_{1}^{\prime}=\left\{r_{1} \ldots r_{m}\right\}$ and $F_{2}^{\prime}=\left\{c_{1} \ldots c_{k}\right\}$.

Definition 5: The costs of the footprints $F_{1}^{\prime}$ and $F_{2}^{\prime}$ are defined as:

$$
\begin{aligned}
& C_{F_{1}^{\prime}}=\max _{r_{i} \in\left\{r_{1} \ldots r_{m}\right\}} M_{1}\left(r_{i}\right) \\
& C_{F_{2}^{\prime}}=\max _{c_{i} \in\left\{c_{1} \ldots c_{k}\right\}} M_{2}\left(c_{i}\right)
\end{aligned}
$$

Definition 6: We define the cost of $F^{\prime}$ as the maximum of $C_{F_{1}^{\prime}}$ and $C_{F_{2}^{\prime}}$.

A high-level pseudocode of our transformation is shown in Figure 1. The ComputeM2 function shows the computation of the map $M_{2}$ according to the definition 4 . The

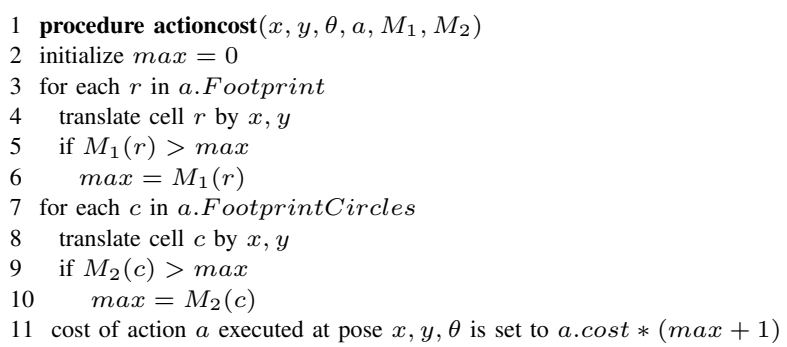

Fig. 2. Evaluation of the cost of the action $a$ executed by the robot at pose $x, y, \theta$. The evaluation uses previously computed maps $M_{1}$ and $M_{2}$ and nominal cost a.cost (i.e., length or execution time) of the action.

PrecomputeActions function performs the transformation of the action footprint $F$ into the new footprint $F^{\prime}$ according to the definitions 2-3. It performs this transformation for every action $a$ in a list of actions for every possible orientation $\theta$ of the robot. Finally, Figure 2 gives the computation of the cost of any action $a$ executed at any pose $x, y, \theta$ according to definitions 5-6. This function is called repeatedly during planning. The function uses maps $M_{1}, M_{2}$ in evaluating the footprint $F^{\prime}$ of action $a$. The footprint is given as the set of circles, a.FootprintCircles, and the set of remaining cells a.Footprint.

\section{TheORETICAL ANALYSis}

In this section we prove the correctness of the proposed method

Theorem 1: Suppose we are given a footprint $F$ with cost $C_{F}$. If we remove circles of radius $r$ from the footprint according to the definitions 1-6 and create a new footprint $F^{\prime}$ with cost $C_{F^{\prime}}$, then $C_{F}=C_{F^{\prime}}$.

\section{Proof}

We call the cell with maximum value in $F f_{p}$. In other words, $C_{F}=M_{0}\left(f_{p}\right)$. After applying our transform we get a new footprint $F^{\prime}=\left\{r_{1} \ldots r_{m}\right\} \cup\left\{c_{1} \ldots c_{k}\right\}$. Definition 2 states that one and only one of the following cases must hold:

1) $D\left(f_{p}, c_{j}\right) \leq r$ for some $j$.

2) $f_{p}=r_{j}$ for some $j$

Case $1-D\left(f_{p}, c_{j}\right) \leq r$ : We first argue that $M_{2}\left(c_{j}\right)=M_{0}\left(f_{p}\right)$ and $C_{F_{2}^{\prime}}=M_{2}\left(c_{j}\right)$. Definition 3 states that all cells within radius $r$ of $c_{j}$ are in the original footprint. Since $f_{p}$ is the maximum of the footprint, $f_{p}$ must be the largest value within radius $r$ of $c_{j}$. By Definition 4, this means that $M_{2}\left(c_{j}\right)=M_{0}\left(f_{p}\right)$. Using these same two rules and following similar logic we can also show that $\nexists k$ such that $M_{2}\left(c_{k}\right)>M_{2}\left(c_{j}\right)$. By Definition 5 this means $C_{F_{2}^{\prime}}=M_{2}\left(c_{j}\right)=M_{0}\left(f_{p}\right)$.

Next we would like to show that $C_{F^{\prime}}=C_{F_{2}^{\prime}}$. This equality will follow from definition 6 if we show that $C_{F_{2}^{\prime}} \geq C_{F_{1}^{\prime}}$. We prove this by contradiction. Assume $C_{F_{2}^{\prime}}<C_{F_{1}^{\prime}}$. Using definition 4 this means that $\exists k$ such that $M_{1}\left(r_{k}\right)>M_{2}\left(c_{j}\right)$. The definition of $M_{1}$ illustrated in part 1 of definition 4 shows that $M_{1}\left(r_{k}\right)=M_{0}\left(r_{k}\right)$. Definition 2 states that $r_{k}$ must be a member of the original footprint. If $r_{k}$ is a 
member of the original footprint then $M_{0}\left(f_{p}\right) \geq M_{1}\left(r_{k}\right)$ because $f_{p}$ is the largest value in the footprint by definition. But we have already shown that $M_{2}\left(c_{j}\right)=M_{0}\left(f_{p}\right)$ and $M_{2}\left(c_{j}\right)<M_{1}\left(r_{k}\right)$. So we have a contradiction. Therefore, $C_{F^{\prime}}=C_{F_{2}^{\prime}}=M_{2}\left(c_{j}\right)=M_{0}\left(f_{p}\right)=C_{F}$.

Case $2-f_{p}=r_{j}$ : First we argue that $C_{F_{1}^{\prime}}=M_{1}\left(r_{j}\right)$ and $M_{1}\left(r_{j}\right)=M_{0}\left(f_{p}\right)$. We show this by contradiction. Assume $C_{F_{1}^{\prime}} \neq M_{1}\left(r_{j}\right)$. By definition 5 this means $\exists k$ such that $M_{1}\left(r_{k}\right)>M_{1}\left(r_{j}\right)$. Definition 2 states that both $r_{j}$ and $r_{k}$ were in the original footprint $F$. But then $r_{j}$ would not be the maximum value in the footprint. This is a contradiction because $r_{j}=f_{p}$ and $f_{p}$ is the maximim value in the footprint by definition. So $C_{F_{1}^{\prime}}=M_{1}\left(r_{j}\right)=M_{0}\left(f_{p}\right)$.

Next we need to show $C_{F^{\prime}}=C_{F_{1}^{\prime}}$. This equality will follow from definition 6 if we show that $C_{F_{1}^{\prime}} \geq C_{F_{2}^{\prime}}$. Again we show this by contradiction. Assume $C_{F_{1}^{\prime}}<C_{F_{2}^{\prime}}$. This means $\exists k$ such that $M_{2}\left(c_{k}\right)>M_{1}\left(r_{j}\right)$. By definition 4, this means that $\exists l$ such that $D\left(m_{l}, c_{k}\right) \leq r$ and $M_{0}\left(m_{l}\right)>M_{0}\left(r_{j}\right)=M_{1}\left(r_{j}\right)$. Definitions 2 and 3 state that $m_{l}$ and $r_{j}$ were inside the original footprint. This means that $r_{j}$ can not be the maximum value in the footprint. This poses a contradiction because $r_{j}=f_{p}$ and $f_{p}$ is the maximum in the footprint by definition. Therefore $C_{F_{1}^{\prime}} \geq C_{F_{2}^{\prime}}$ and $C_{F^{\prime}}=C_{F_{1}^{\prime}}$ by definition 6 . Thus $C_{F^{\prime}}=M_{1}\left(r_{k}\right) \stackrel{2}{=} M_{0}\left(f_{p}\right)=C_{F}$.

\section{INTEGRATION WITH PLANNER}

We have integrated our method into a lattice-based planner [10][14]. Lattice-based planning allows for planning complex dynamically-feasible maneuvers. A state lattice [14] is a discretization of the configuration space into a set of states, representing configurations, and connections between these states, where every connection represents a feasible path. As such, lattices provide a method for motion planning problems to be formulated as graph searches. However, in contrast to many graph-based representations (such as 4connected or 8-connected grids), the feasibility requirement of lattice connections guarantees that any solutions found using a lattice will also be feasible. This makes them very well suited to planning for non-holonomic and highlyconstrained robotic systems, such as passenger vehicles.

In our scenario, we define each state to be a 3-dimensional state: $x, y, \theta$. To demonstrate the benefits of our proposed optimization, we consider a square robot capable of turning in place as well as moving forward while rotating. (An example of actions suitable specifically to large non-holonomic vehicles can be found in [10]). Fourteen actions are used in our lattice, each defined by a unique combination of forward velocity, rotational velocity and time. We define two turnin-place actions, one for turning left forty-five degrees and another for turning right by the same amount. We define five forward actions with a duration of one second and a forward velocity of $1 \mathrm{~m} / \mathrm{s}$. The rotational velocity varies for each action but remains constant throughout the execution of the action. The range of rotational velocities covers $-\pi / 2$ to $\pi / 2$. Additionally, we define five identical backward actions. For these, the forward velocity is defined to be $-1 \mathrm{~m} / \mathrm{s}$. Finally, we define an extra forward and backward action with a shorter duration than the others to allow for the robot to move forward or backward by a single cell. These actions have no rotational velocity. Figure 3 shows the trajectories of all of the actions. The two turn in place actions coupled with the short forward and backward action guarantee that the planner will generate a path when one is available. The ten longer actions allow for the planner to select actions which make larger progress toward the goal, thereby reducing the overall length (in terms of number of actions) of the planned path. Note that our method could be used with a nonholonomic vehicle by adjusting the action space to include only actions feasible for the robot given its constraints.

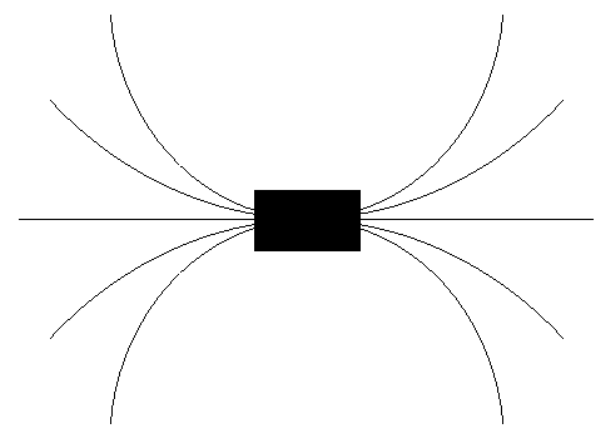

Fig. 3. Action Space: Pictured are ten of the fourteen predefined actions in the action space. Each action is uniquely defined by a translational veloctiy, rotational velocity and duration. In addition to each of these actions, the action space contains four more predefined actions which cannot be seen in the figure: turn in place left, turn in place right, one short forward action and one short reverse action. The short actions allow for movement by a single grid space.

During the pre-computation step, we determine the set of states covered by the robot as it executes each action. We then perform the transformation defined above on the state set. To do this, we fit a set of circles inside the footprint of the robot as it stands still. We then translate these circles along the path of the robot, rotating them as the body of the robot rotates through the action. This sweeps a set of points from the original footprint and leaves only the set of points that did not fall inside a circle at any point during the action. These become the remainder set. We record the locations of the circle centers as they translate and rotate through the action. These points become the center set. Figure 4 shows the footprints of the actions before and after the transformation. Note that only five actions are pictured in the figure. Each of the fourteen predefined actions is a rotation or reflection (or both) of one of these five actions.

To search the constructed lattice-based graph, we use ARA* search [9]. ARA* exploits a property of $A *$ that can result in much faster generation of solutions, namely that if consistent heuristics are used and multiplied by an inflation 


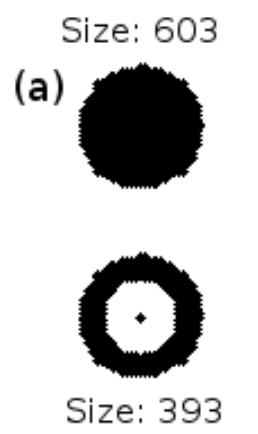

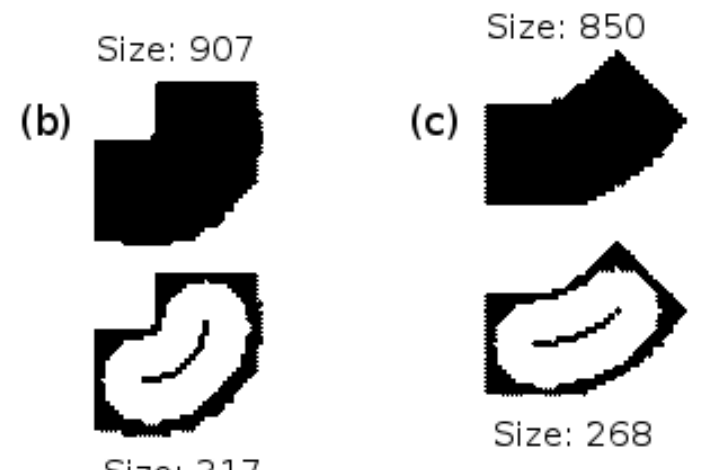

Size: 317
Size: 462

(d)
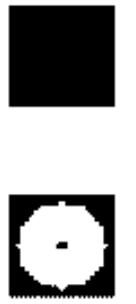

Size: 193
Size: 840

(e)
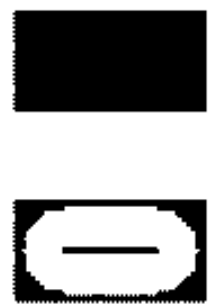

Size: 247

Fig. 4. Action Footprints before and after transformation: This figure shows the footprint of the robot as it executes each action. The number of cells in the footprint is indicated for each action. This number directly corresponds to the number of points involved in the convolution with the cost map. Each of the fourteen actions is a rotation or reflection of one of the five actions pictured above.

factor $\epsilon>1$, then $\mathrm{A}^{*}$ can often generate a solution much faster than if no inflation factor is used [6], and the cost of the solution generated by $\mathrm{A}^{*}$ will be at most $\epsilon$ times the cost of an optimal solution [4]. ARA* operates by performing a series of these inflated $A^{*}$ searches with decreasing inflation factors, where each search reuses information from previous searches. By doing so, it is able to provide suboptimality bounds on all solutions generated and allows for control of these bounds, since the user can decide how much the inflation factor is decreased between searches.

\section{EXPERIMENTAL ANALYSIS}

We utilize two separate test scenarios. In the first scenario, we allow the planner enough time to compute the optimal path and compare the amount of time to run with and without the transformation detailed above. In the second test, we limit the amount of time the planner is given to compute a path and compare the cost of the paths achieved by the planner when run with and without our optimization. This test more accurately represents the use of a system in the real world, where planning time is often limited.

We define two environments for our testing. The first is a $25 \mathrm{~m}$ by $25 \mathrm{~m}$ space discretized into a 500 by 500 grid with each cell representing a $5 \mathrm{~cm}$ by $5 \mathrm{~cm}$ area. We define the robot to be $1 \mathrm{~m}$ by $1 \mathrm{~m}$. The second environment is a
$48 \mathrm{~m}$ by $54 \mathrm{~m}$ space discritized into cells of size $2.5 \mathrm{~cm}$ by $2.5 \mathrm{~cm}$. In this environment, the robot is defined as a $0.5 \mathrm{~m}$ by $0.5 \mathrm{~m}$ square. Figure 5 shows the environments.

All times presented in the following result sets were recorded from runs of the software executed on a MacBook Pro with a Pentium Dual Core processor.

\section{A. Optimal Planning}

For the first set of tests we utilize the planner with and without our proposed cost computation optimization. Our main goal for these tests is to study the effectiveness of the algorithm. We select three different start and goal locations for each environment. The planner is run for each different start/goal pair and we average the results across runs. Table I shows the results. We study two measurements of time, the time to precompute and the time to plan. The time to precompute includes the time it takes to generate the action footprints pictured in figure 4, bottom images. It also includes the time it takes to generate the map $M_{2}$ using the rules defined in the Algorithm section of this paper. 


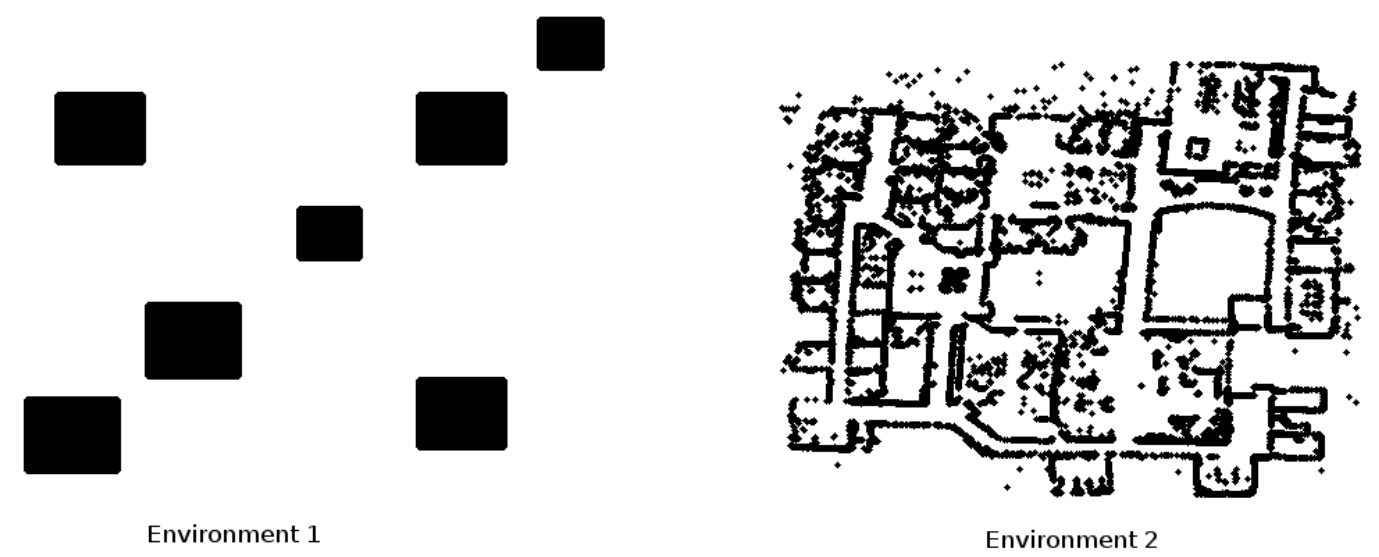

Fig. 5. Test Environments: The figures above show two test environments.

\begin{tabular}{|c|cc|cc|}
\hline \multirow{2}{*}{ World } & \multicolumn{2}{|c|}{ Without Optimization } & \multicolumn{2}{c|}{ With Optimization } \\
& Time To Precompute(s) & Time To Plan(s) & Time To Precompute(s) & Time To Plan(s) \\
\hline $1-1$ & .460 & 8.410 & .670 & 2.880 \\
$1-2$ & .450 & 23.380 & .660 & 9.790 \\
$1-3$ & .430 & 23.450 & .660 & 9.810 \\
$2-1$ & .690 & 696.480 & 1.090 & 116.760 \\
$2-2$ & .740 & 156.270 & 1.150 & 24.970 \\
$2-3$ & .740 & 1001.00 & 1.170 & 81.690 \\
\hline
\end{tabular}

TABLE I

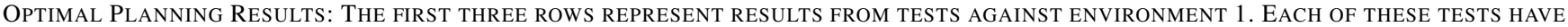
A UNIQUE START AND GOAL STATE. THE SECOND THREE ROWS REPRESENT RESULTS FROM TEST AGAINST ENVIRONMENT 2.

(a)

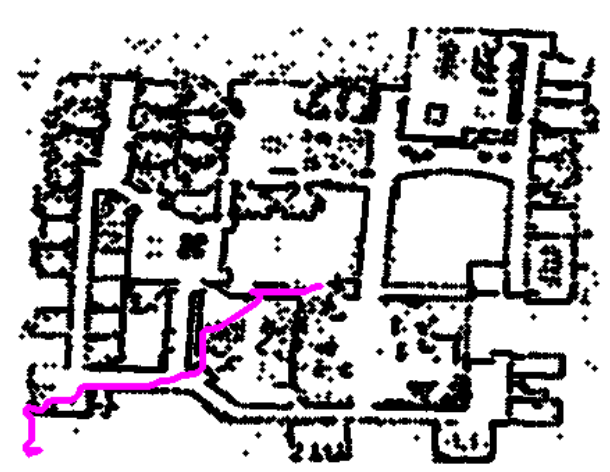

(b)

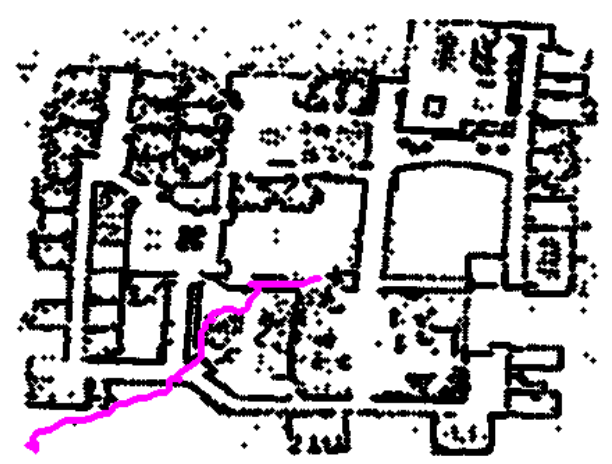

Fig. 6. Efficient Path Computation: The figure above shows the path planned by the planner when given 4.0 seconds to produce a solution. the figure on the left shows the path produced without using our transformation. The figure on the right shows the path produced using the optimization.

\section{B. Real-time Planning}

For the second set of tests, we examine the performance of the planner in real-time environments where suboptimal planning is often necessary to meet real-time requirements. We consider a single environment with a single start/goal location pair. Specifically, we select the configuration which took the largest amount of time to achieve an optimal path in the first set of tests. We vary the time limit on the planner and compare the optimality of the planned paths. Table II shows the results of this set of tests. Figure 6 compares the paths acheived when planning time is limited to four seconds for each planner.

To show the statistical soundness of our results we run a final set of tests. Here we run the planner multiple times, randomly selecting a start and goal for each run. We examine the percentage increase in precomputation time and the percentage decrease in planning time when using our 


\begin{tabular}{|c|cc|cc|}
\hline & \multicolumn{2}{|c|}{ Without } & Optimization & \multicolumn{2}{c|}{ With Optimization } \\
Time(s) & Cost & Epsilon & Cost & Epsilon \\
\hline 0.5 & - & - & 51000 & 5.0 \\
1.0 & - & - & 51000 & 4.4 \\
2.0 & 51000 & 5 & 51000 & 3.8 \\
4.0 & 51000 & 5 & 48000 & 2.2 \\
8.0 & 48000 & 2.2 & 47000 & 1.4 \\
16.0 & 44000 & 1.6 & 40000 & 1.2 \\
\hline
\end{tabular}

TABLE II

Real-time Planning Results: This table shows Results PRODUCED WHEN PLANNING TIME IS LIMITED. THE NON-OPTIMIZED

VERSION OF THE PLANNER FAILS TO FIND A SOLUTION UNTIL GIVEN AT

LEAST 5.0 SECONDS TO PLAN. ADDITIONALLY, THE OPTIMIZED

VERSION OF THE PLANNER CONSISTENTLY OUTPERFORMS THE NON-OPTIMIZED VERSION IN BOTH COST OF THE RESULTANT PATH AND ACHEIVED EPSILON VALUE.

\begin{tabular}{|c|cc|}
\hline World & Precomputation Time Increase (\%) & Plan Time Savings (\%) \\
\hline 1 & 58.60 & 63.00 \\
\hline
\end{tabular}

TABLE III

Computation Time Savings: The table shows the aVerage $\%$

INCREASE IN PRECOMPUTATION TIME AND THE \% DECREASE IN

PLANNING TIME WHEN PLANNING BETWEEN RANDOM START AND GOAL

LOCATIONS IN EACH ENVIRONMENT.

transformation. Results are averaged across runs. Table III shows the results when we give the planner enough time to calculate the optimal path.

\section{CONCLUSIONS}

Our experimental results show around a sixty percent decrease in planning time when using the transformation we propose. This savings is a savings that will be experienced each time the planner is run. We note that the results also indicate a sixty percent increase in precomputation time. While initially this number may appear to offset any gains made by the planner, it is important to remember that most of the precomputations (precomputing actions) must only be run once at startup. They do not need to be run every time the planner is called. Additionally, the run time of the precomputation step is dependent upon the size and complexity of the robot while the runtime of the planner is dependent upon the size and complexity of the configuration space. Thus, as environments become larger and more densely packed with obstacles the increase in precomputation time becomes small compared to the savings in planning time.

\section{REFERENCES}

[1] M.D.C. Amezquita Benitez, K.K. Gupta, and B. Bhattacharya. Eodm-a novel representation for collision detection. In Robotics and Automation, 2000. Proceedings. ICRA 'O0. IEEE International Conference on, volume 4, pages 3727-3732 vol.4, 2000.

[2] J. Buhmann, W. Burgard, A.B. Cremers, Dieter Fox, T. Hofmann, F. Schneider, J. Strikos, and Sebastian Thrun. The mobile robot rhino. AI Magazine, 16(1), 1995.

[3] Howie Choset, Kevin M. Lynch, Seth Hutchinson, George Kantor, Wolfram Burgard, Lydia E. Kavraki, and Sebastian Thrun. Principles of Robot Motion: Theory, Algorithms, and Implementations (Intelligent Robotics and Autonomous Agents). The MIT Press, June 2005.
[4] H. W. Davis, A. Bramanti-Gregor, and J. Wang. The advantages of using depth and breadth components in heuristic search. In Z. W. Ras and L. Saitta, editors, Methodologies for Intelligent Systems, 3, pages 19-28, New York, 1988. North-Holland.

[5] A. Elfes. Using occupancy grids for mobile robot perception and navigation. Computer, 22(6):46-57, 1989.

[6] John Gary Gaschnig. Performance measurement and analysis of certain search algorithms. PhD thesis, Carnegie Mellon University, 1979.

[7] M.H. Hassoun. Fast computation of optimal paths in two- and higher dimension maps. In IEEE International Symposium on Circuits and Systems, 1990.

[8] Steven M. LaValle. Planning Algorithms. Cambridge University Press, New York, NY, USA, 2006.

[9] M. Likhachev, G. Gordon, and S. Thrun. ARA*: Anytime A* with provable bounds on sub-optimality. In Advances in Neural Information Processing Systems. MIT Press, 2003.

[10] Maxim Likhachev and Dave Ferguson. Planning long dynamicallyfeasible maneuvers for autonomous vehicles. In Proceedings of Robotics: Science and Systems IV, Zurich, Switzerland, June 2008.

[11] Ming Chieh Lin. Efficient collision detection for animation and robotics. PhD thesis, University of California, Berkeley, 1993. ChairCanny, John F.

[12] T. Lozano-Perez. Spatial planning: A configuration space approach. Computers, IEEE Transactions on, C-32(2):108-120, Feb. 1983.

[13] M. Nakamiya, Y. Kishino, T. Terada, and S. Nishio. A route planning method using cost map for mobile sensor nodes. In International Symposium on Wireless Pervasive Computing, 2007.

[14] Mikhail Pivtoraiko and Alonzo Kelly. Generating near minimal spanning control sets for constrained motion planning in discrete state spaces. In Proceedings of the 2005 IEEE/RSJ International Conference on Intelligent Robots and Systems (IROS '05), pages 3231 - 3237, August 2005.

[15] J. Tornero, J. Hamlin, and R.B. Kelley. Spherical-object representation and fast distance computation for robotic applications. In Proceedings of IEEE International Conference on Robotics and Automation, Sacramento, California, April 1991.

[16] Hong Yang, Johann Borenstein, and David Wehe. Sonar-based obstacle avoidance for a large, non-point, omni-directional mobile robot. In Omni-directional Mobile Robot, Spectrum 2000 International Conference on Nuclear and Hazardous Waste Management, pages 2428, 2000. 